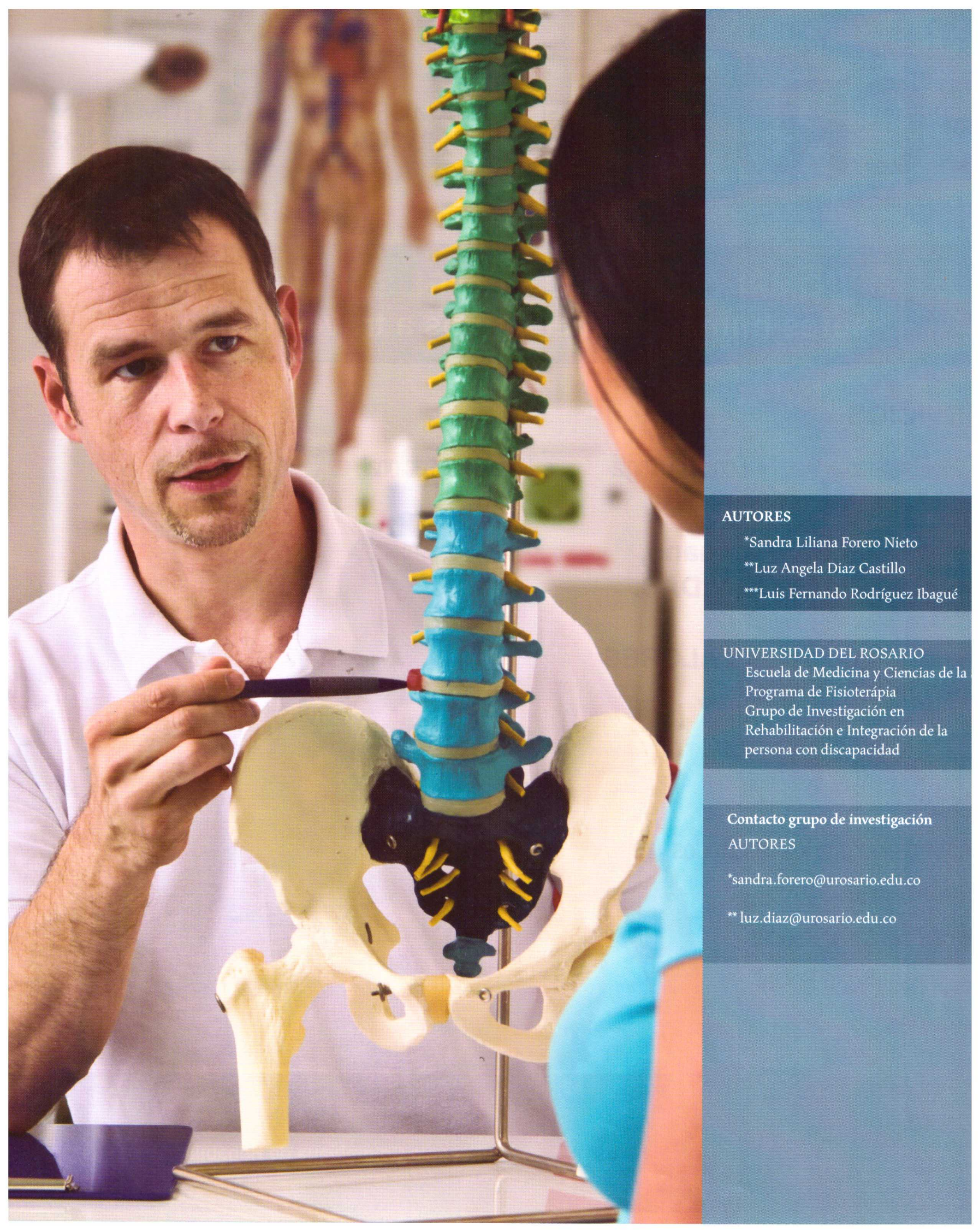




\section{RAZONAMIENTO CLÍNICO EN FISIOTERAPIA}

Fecha de recepción 17 de Agosto de 2010 . Fecha de Aprobación 22 de Septiembre de 2010

\section{RESUMEN}

El presente artículo muestra los resultados de la primera fase del proyecto de investigación titulado: "Diferenciación en el razonamiento clínico de estudiantes, novatos y expertos en el campo de la Rehabilitación Humana" el cual se desarrolla en la Red hospitalaria Méderi, a cargo de los autores del presente documento. Para esta fase se realizó un muestreo intencionado de la categoría "razonamiento clínico" cuyos criterios de inclusión para la selección de la muestra fueron: documentos publicados en diferentes épocas, revisión de publicaciones en revistas indexadas de las bases de datos Science Direct, Ovid, Medline y libros publicados que contuvieran temas relacionados con el objeto de esta revisión a nivel local, nacional e internacional. El total de la muestra fue de 80 documentos, de los cuales 15 corresponden a capítulos de libro y 60 a artículos de revista. Todos los documentos fueron revisados y analizados sistemáticamente a través de la estrategia de reseñas críticas. Posteriormente, se realizó el cotejo de la información, mediante el análisis narrativo de contenido, lo que permitió identificar los significados comprendidos en la muestra.

PALABRAS CLAVE

Razonamiento clínico, clínica, terapéutica.

\section{ABSTRACT}

This paper shows the results of the first phase of the research project entitled: "Differentiation in the clinical reasoning of students, beginners and experts in the field of Human Rehabilitation" which takes place in the Mederi hospital network, conducted by the authors of this paper. For this phase, we sampled more intentional "clinical reasoning, " whose inclusion criteria for the selection of the sample were: documents published at different times, review of publications in journals indexed in Science Direct database, Ovid, Medline and books that contain themes related to the subject of this review locally, nationally and internationally. The total sample consisted of 80 documents, of which 15 correspond to book chapters and 60 to journal articles. All documents were reviewed and analyzed systematically through the strategy of critical reviews. Finally, we reviewed all the collected information through the narrative content analysis, which identified the meanings in the sample.

KEY WORDS

Clinical reasoning, clinical enviromment, therapeutic. 
$\mathrm{L}$ os fisioterapeutas han experimentado cambios importantes en el ejercicio y visión de su quehacer, impulsados por los marcos normativos, la comprensión del objeto de estudio, y las preguntas e intereses formulados en torno a los modelos de atención, la epistemología, la explosión del conocimiento, la globalización y el desarrollo profesional (1).

Estas realidades e interrogantes han influido en la forma como se aborda el objeto de estudio, los alcances de la práctica de la disciplina, las características y las habilidades necesarias en el profesional para el buen desarrollo de su ejercicio laboral, el potencial de sus graduandos y las competencias que deben ejercitarse en el proceso de formación del fisioterapeuta para la buena praxis. Todas ellas exigentes de un alto nivel intelectual y un alto nivel de experticia técnica que llevan a la autonomía en la práctica y regulación de la disciplina, el compromiso público de $\operatorname{servicio}(2,3)$ y la capacidad de tomar decisiones de la más alta calidad con el propósito de obtener los mejores resultados posibles frente a una situación profesio$\operatorname{nal}(5,6,7,8)$.

La toma de decisiones requiere para su materialización procesos de razonamiento complejos que involucran el análisis de múltiples variables, diversas probabilidades y posibles consecuencias, así como la identificación de propuestas resolutivas o atenuantes del problema presente, teniendo en cuenta aspectos confluyentes e interdependientes $(4)$ de orden personal (sobre el caso en consideración y del terapeuta), aspectos derivados de la incertidumbre y aspectos contextuales del ambiente y la cultura que juegan un importante papel en la interacción profesional.

El razonamiento es considerado como el proceso consciente y lógico en el que se utiliza la razón para establecer conceptos encaminados a demostrar algo o a persuadir a oyentes y lectores(9) donde el razonador es consciente de que un juicio- la conclusión-, es determinada por otro juicio(10) que valida o falsea la hipótesis planteada en una situación determinada e involucra conocimientos aprendidos y/o experiencias vividas.

La clínica como concepto aparece en la historia de la formación médica a mediados de 1658, como el estudio de la aplicación de técnicas específicas independientes del estudio básico de la patología, de manera tal que la aplicación de estas artes tenía una jerarquía de diferente rango(11). Es sólo hasta finales del siglo XX cuando se integra la patología y la clínica a la enseñanza de los estudiantes, otorgando igual importancia a las ciencias básicas y a las asignaturas clínicas en los esquemas curriculares de la época (12). Las escuelas francesas dan con ello el paso a la clínica moderna, considerada hoy un compuesto dinámico que involucra y conjuga los componentes de aprendizaje teórico de las ciencias básicas y la patología: (cuerpo teóríco), la aplicación e integración de técnicas (procedimientos) y la práctica supervisada $(13)$ (Ver figura 1$)$.

En el marco de estos referentes, surge el razonamiento clínico en fisioterapia como un complejo proceso cognitivo que tiene lugar en el escenario y la experiencia de la clínica, donde se hace frente: problemas de naturaleza patológica que afectan o pueden afectar la estructuras y funciones corporales (deficiencias), las actividades (li mitación en la actividad) y la participación(restricción en la partici pación) (14). Este razonamiento es pieza fundamental en la práctici profesional en el área en la medida que permite ejercer y demostra la responsabilidad, la competencia y la autonomía profesional; ayu da a preservar los conocimientos a lo largo de la vida y contribuye a desarrollo del objeto de estudio disciplinar. $(15,16)$. Sin la presenci. de este proceso la práctica clínica se convierte en una operación d nivel técnico que requiere la dirección y supervisión de un tomado de decisiones que defina procesos evaluativos, diagnósticos, pronós ticos, conductas y reevaluación $(17,18)$.

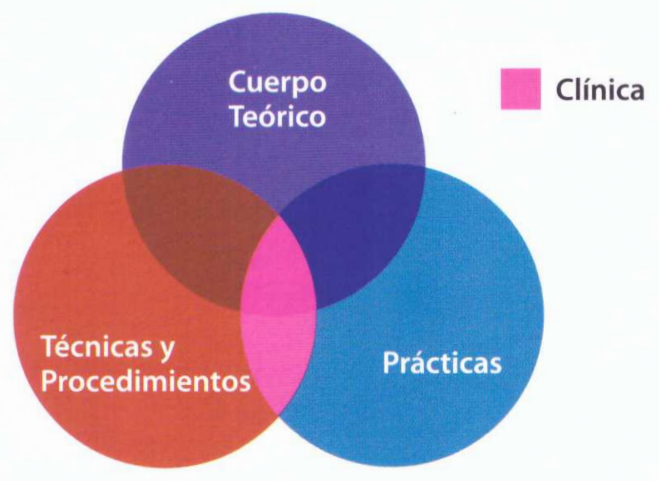

Fig. 1. Componentes actuales de la clínica

Los juicios que sirven como punto de partida para este procesc se denominan premisas(19) y desempeñan la función de ser las condiciones del razonamiento que, en términos generales, tiene una estructura conformada por tres grandes elementos: el conocimiento e información disponibles, los procesos de pensamiento y las inferencias formadas (20); que implican el génesis de distintos modelos de razonamiento.

A la luz del marco anteriormente planteado el razonamiento clínico se concibe como una destreza crítica que utiliza el proceso cognitivo(21) para la toma de decisiones profesionales en relación con la planificación, dirección, realización y reflexión del proceso evaluativo, la formulación de un diagnóstico, el plan de tratamiento, metas a corto, mediano y largo plazo, los riesgos previsibles y el pronóstico funcional del usuario $(21,22)$, en donde a través de un continuum metodológico se obtienen las mejores conclusiones en una situación del ámbito clínico(23), siempre cimentado en destrezas de pensamiento crítico necesarias para el buen desempeño disciplinar.

Se han definido seis pasos para la toma de decisiones como flujo interdependiente (ver Figura 2) y organizado(24) que como se expondrá más adelante, se convierten en sí mismos en un tipo de razonamiento inserto en el flujo de acciones que deben ser conocidas y entrenadas para el logro de habilidades en el ejercicio de razonar. 


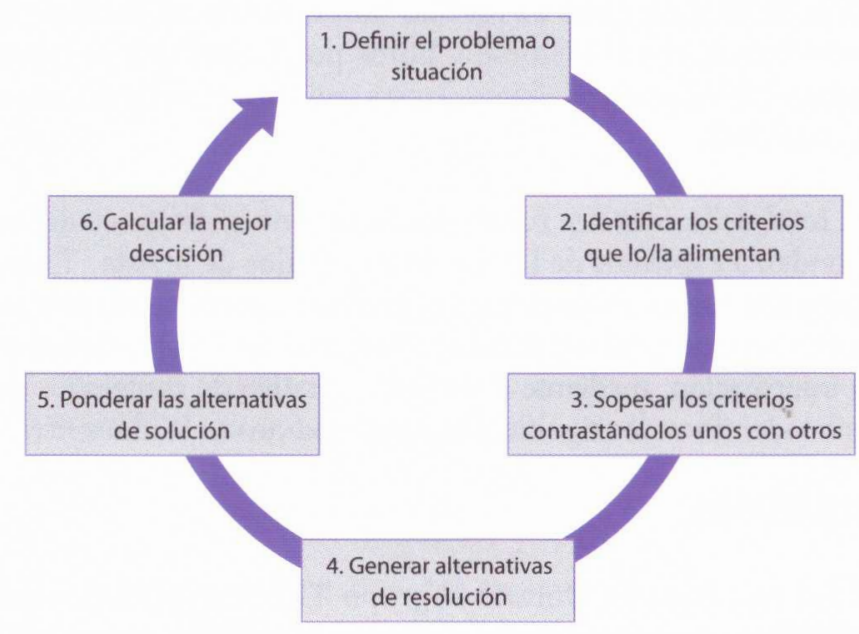

Fig. 2. Flujo de acciones en la toma de decisiones

Son varias las teorías de toma de decisiones propuestas por diferentes ciencias tanto naturales como económicas y exactas, con el propósito de encontrar un solo modelo unificador que hasta hoy no ha sido posible determinar. Offredy y col (23) agrupan las diversas teorías en tres grandes grupos:

1. Teoría de análisis de la decisión, también llamada prescriptiva o normativa, involucra modelos estadísticos e incluye el modelo bayesiano. Esta proporciona una visión prescriptiva del proceso de la toma de decisiones.

2. Teoría del comportamiento ante la decisión: incluye el análisis de premisas sociales y se utiliza comúnmente como método experimental para observar el proceso de toma decisiones en escenarios reales.

3. Teoría del procesamiento de la información: utiliza la visión aproximada del proceso con el reporte verbal de datos acerca de los procesos que se llevan a cabo.

La primera de estas teorías se enfila en una visión prescriptiva de la toma de decisiones, mientras que las dos últimas hacen énfasis en las aproximaciones descriptivas de este proceso y se utilizan en los estudios que pretenden dar cuenta de los mecanismos mentales que se entrelazan para razonar.

Las tres teorías enunciadas utilizan herramientas angulares básicas (ver figura 3) para explicar los supuestos que las constituyen, refiriéndose a capacidades cognitivas básicas, habilidades de procesamiento cognitivo individual y disposición y estilo de pensamiento $(24,25)$. Ellas pueden ser generalizadas (como indispensables) para el abordaje de todo problema o contexto situacional, anticipando efectos indeseables o deseables (26). Veamos ahora la determinación clásica de éstas 3 herramientas de razonamiento:

Conocimiento: Desde Platón y Aristóteles (400-30o AdC) que lo consideraban como episteme, techne y phronesis, el conocimiento se ha establecido en dos maneras: el científico (de la creatividad y la producción) y el práctico (de la sabiduría y la ética) haciendo referencia al "conocer el como" y a "conocer de esto" $(27,28)$. En términos generales hace referencia a la capacidad humana de resolver un conjunto determinado de problemas con cierta efectividad. Contiene dos acepciones: una estática equivalente a saber y otra dinámica que indica un proceso del saber a no saber (29). Según Piaget el conocimiento es una construcción que se da entre la interacción de los sujetos cognoscentes y los objetos cognoscibles (30). La psicología constructivista lo define también como una construcción deliberada de los seres humanos que se realiza a través del esfuerzo por entender la naturaleza y la experiencia(18).

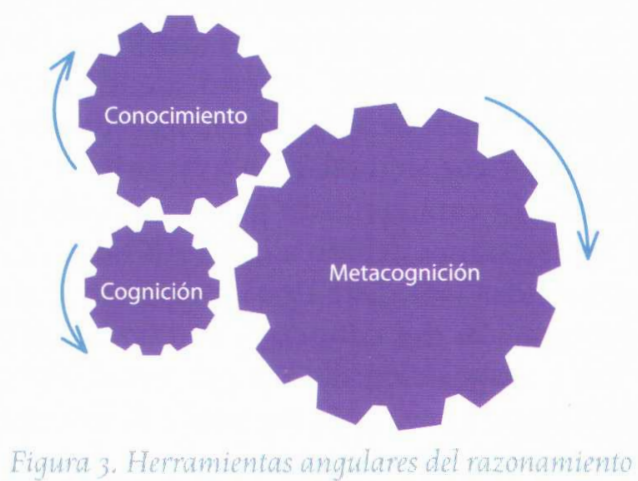

Cognición: Este término hace referencia al uso, adquisición y mantenimiento del conocimiento(31) e incluye aspectos individuales y grupales determinados por la interacción con otras personas (comunicativa), el conocimiento individual (mental) y la interacción con los objetos(distribuida). Incluye la percepción de información relevante, la interpretación de datos específicos o inducción, la deducción para generar hipótesis y la capacidad de probarlas para afirmarlas o declararlas falsas.

Metacognición: Es un término que abarca diversas habilidades humanas tales como la metacomprensión, el automonitoreo, el seguimiento metacognitivo y el autoaprendizaje. Diversos estudios demuestran que las personas con mayores habilidades metacognitivas tienen mayor capacidad para enfrentarse a tareas nuevas, mayor destreza oral y escrita y pericia para seguir los pasos para resolver un problema.(32) De manera que la metacognición hace referencia a la capacidad humana de pensar y reflexionar sobre los trancursos cognitivos que son desplegados en la acción de toma de decisiones. Es el pensamiento reflexivo y crítico que debe incluir la información disponible, el proceso de razonamiento, las hipótesis formuladas y las decisiones tomadas con base en la evidencia científica y la experiencia, la organización y contenido del conocimiento profesional y personal $(33,21)$.

\section{Modelos de razonamiento clínico}

Los estándares de práctica, los modelos epistemológicos y la comprensión del hacer y del pensar del fisioterapeuta han modificado las interpretaciones y estrategias del razonamiento clínico. Algunos de los modelos acá presentados han sido apropiados por la fisioterapia en diferentes tiempos y situaciones, a la vez que han modelado su historia disciplinar (34). 
Los modelos basados en el paradigma positivista corresponden a modos de pensamiento científico. El modelo hipotético deductivo $(34,35)$ : es un modelo derivado de la investigación médica en el que la generación de hipótesis se basa en los datos clínicos obtenibles y el conocimiento del profesional. Esta generación de hipótesis se halla enmarcada en un pensamiento de tipo inductivo donde se realiza un procesamiento de lo específico a lo general y deductivo en el cual las inferencias se realizan de lo general a lo específico. En este modelo el razonamiento inductivo genera las hipótesis y el deductivo las analiza para determinar procedimientos de atención. Aunque pertenece a las ciencias cógnitivas, tiene su asiento en el paradigma empírico-analítico. En el Patrón de reconocimiento el razonador experto identifica en situaciones problemáticas patrones de semejanza, recordación o de buenos conocimientos teóricos, con relación a experiencias previas o "patrones de libro". Es caracterizado por la velocidad y la eficiencia en la toma de decisiones debido a la sensibilidad ante situaciones vividas o aprendidas (36). Este tipo de razonamiento se completa con el de integración del conocimiento donde el clínico utiliza la ordenación y disposición del conocimiento almacenado en la memoria para llegar a conclusiones, desde varias hipótesis.

Gracias al esfuerzo por convertir a la fisioterapia en disciplina, se han apropiado y diseñado metodologías, modelos e instrumentos propios del fisioterapeuta. El paradigma biopsicosocial de la salud ha ejercido un importante moldeamiento a los procesos de salud-enfermedad y discapacidad(37,38), propios de la clínica. La teoría del movimiento como continuum y la teoría del movimiento como sistema complejo(39,40), abordan al usuario como un ser con implicaciones multidimensionales desde lo microscópico a lo macroscópico y desde la interacción del control motor, aprendizaje motor y el contexto respectivamente. Estas teorías muestran la evolución del objeto de estudio e indican que los modelos asentados en el positivismo, si bien son fundamentales y eficaces, son insuficientes para la toma de decisiones asertiva(41) en la medida que se reconoce al usuario como actor protagonista del proceso razonador, donde la autopercepción del paciente de su problema, la capacidad de asumirlo de forma activa y no pasiva y las habilidades de participación(42) son la clave de la recuperación, mejoría o mantenimiento de su nivel de salud.

\section{METODOLOGIA}

El presente artículo muestra los resultados de la primera fase del proyecto de investigación titulado: "Diferenciación en el razonamiento clínico de estudiantes, novatos y expertos en el campo de la Rehabilitación Humana" el cual se desarrolla en la Red hospitalaria Méderi, a cargo de los autores del presente documento.

Para su elaboración, se realizó una revisión documental, que permite conocer el marco teórico que contextualiza esta problemática (5).

Para esta fase se realizó un muestreo intencionado de la categoría "razonamiento clínico" (6) cuyos criterios de inclusión para la selección de la muestra fueron: documentos publicados en diferentes épocas, revisión de publicaciones en revistas indexadas de las bases de dato Science Direct, Ovid, Medline y libros publicados que contuvierar temas relacionados con el objeto de esta revisión a nivel local, naciona e internacional.

El total de la muestra fue de 80 documentos, de los cuales 15 co rresponden a capítulos de libro y 60 a artículos de revista. Todos lo documentos fueron revisados y analizados sistemáticamente a travé de la estrategia de reseñas críticas (7). Posteriormente, se realizó el cotej de la información, mediante el análisis narrativo de contenido, lo qur permitió identificar los significados comprendidos en la muestra (8).

\section{RESULTADOS}

En el año 2000 en Ontario el grupo The Provincial Rehabilita tion Reference propone la creación de un modelo para rehabilita ción centrado en el usuario, impulsando el empoderamiento ds las personas con discapacidad y abandonando el "paternalismo" dt los terapeutas (43). Este modelo supone la articulación de una serie d discursos y acciones ajustados a las necesidades de los usuarios par: facilitar el nivel de participación y empoderamiento en el tratamiento (44). Luego de la revisión teórica, los autores proponen la aprehensiór y el reconocimiento del proceso de razonamiento holístico denominadc modelo de razonamiento clínico centrado en el usuario propuesto por Higgs y Jones $(21,28,45)$, para la formación y guía de los fisioterapeutas.

El modelo de razonamiento clínico centrado en el usuario (fig 4) es un modelo colaborativo en donde el usuario y su familia juegan un importante rol en la toma de decisiones y en los resultados finales $\mathrm{d} \epsilon$ la interacción profesional (46). Las variables que influyen en el éxitc de este modelo incluyen: 1 . Los atributos personales y profesionales del fisioterapeuta: sus habilidades comunicativas ( 47 ) y de educación en salud, la calidad y profundidad de los conocimientos, la organización mental de los mismos (48), la experticia $(21,36)$ y las destrezas profesionales; 2. Los atributos del usuario y/o familia establecidos a través de las demandas, necesidades y expectativas manifestadas a través del proceso de interacción, las creencias, el bagaje cultural e intelectual, el nivel físico, el nivel psicosocial y la capacidad de asumir la aceptación o rechazo de los planes de atención (consentimiento informado) y 3 . Los atributos del ambiente representados en el sistema de seguridad social, las políticas y el marco normativo y los contextos culturales, económicos y sociales que puedan influenciarle $(49,50)$.

Para comprender el problema entendido como la diferencia entre una situación real y la esperada, no es suficiente el diagnóstico médico, la fisiopatología y los conceptos patognomónicos de la enfermedad $(21,47,49)$. Es necesario analizar otros factores que influyen en la condición de salud, como el efecto de la deficiencia en el nivel de bienestar y la forma como esa deficiencia es vista y asumida por el implicado. Esta cadena inicia con la percepción que el fisioterapeuta obtiene al observar el usuario y el análisis interpretativo que hace de ella. Esta observación es una destreza importante que debe ser entrenada y guiada ya que es el prerrequisito para resolver el problema e iniciar la cascada de razonamiento y la toma de decisiones $(51,52)$. 

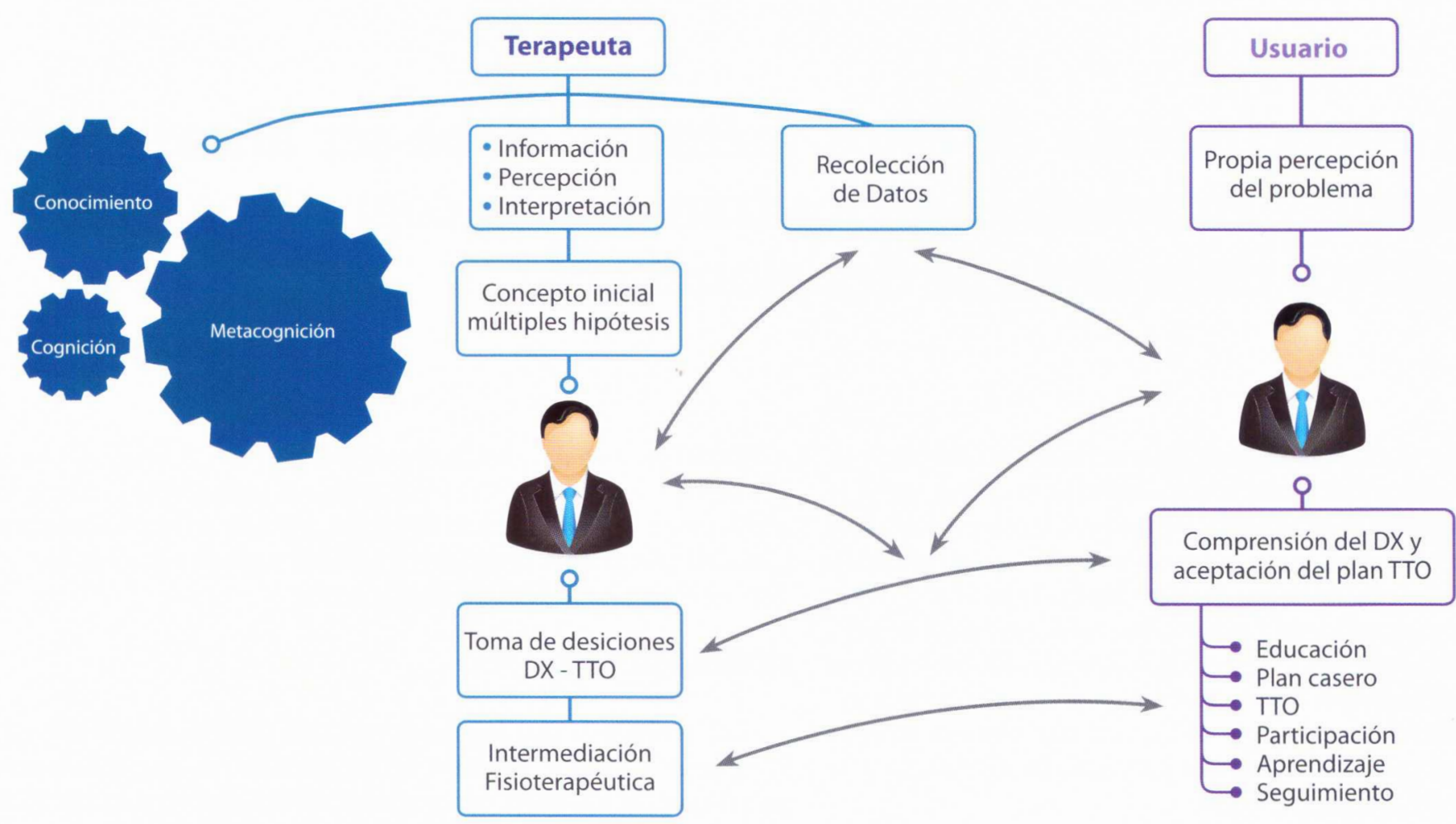

Figura 4. Modelo centrado en el usuario.2004. Adaptado de Jones M, Rivett D.

El siguiente eslabón de este proceso es la recolección de datos que se lleva a cabo a través de la entrevista y el examen físico donde se utilizan instrumentos, herramientas y baterías evaluativas (53). En la entrevista existen preguntas de rutina que deben ser aclaradas para comprender factores ambientales y de aspecto personal del usuario y que deben integrarse con la percepción propia del usuario acerca de su problema(54). Las preguntas claves para determinar estos factores inician con el perfil personal, la ocupación, el aspecto sociobiográfico y familiar, el cuidador o los cuidadores, el familigrama y motivo de consulta, seguido de un análisis retrospectivo que incluya antecedentes, factores de riesgo y protectores, un tamizaje del estado psicológico, cognitivo y afectivo, las demandas y expectativas y el grado de escolaridad.

Es importante también recoger información acerca de las variables socioeconómicas y demográficas que inciden en el usuario tales como sitio de vivienda, seguridad social, redes de apoyo y acceso a servicios públicos. Preguntas puntuales sobre la localización y comportamiento de los signos y síntomas referidos por el usuario, el estado general de salud y la cronología del evento patológico también hace parte de la entrevista.

En el examen físico es necesario ajustar los parámetros evaluativos de acuerdo a la hipótesis planteada inicialmente y la recaudación de los datos suministrados por la entrevista, así como las capacidades propias del usuario, su nivel cognitivo y su percepción sensorial. Seguido a esto se modifica o ratifica la hipótesis y se establece un diagnóstico, el cual se considera como uno de los más importantes resultados del proceso de razonamiento y debe incluir una hipótesis inicial, un diagnóstico diferencial, los test necesarios para retroalimentar la recolección de datos, la priorización de los síntomas y signos que el usuario presenta(55), un pronóstico basado en el análisis prospectivo y el plan de acción fisioterapéutico. Este se explica al usuario y/o familia con el propósito de establecer metas conjuntas, explicitar los riesgos previsibles, los alcances del tratamiento, y obtener un consentimiento informado acerca del proceso de toma de decisiones.

En este aspecto es necesario contar con la educación en salud como estrategia de adherencia al tratamiento, el programa de refuerzo elaborado y dirigido de forma personal al usuario y/o familia y las estrategias de empoderamiento del cuidado de la salud.

La reevaluación y el seguimiento a posteriori de los objetivos planteados y conseguidos, y de ser necesario, la reformulación del plan de acción, es el final y el comienzo del flujograma de acciones del razonamiento. En ella se soportan los signos e intervenciones que se deben modificar o se identifican los datos faltantes o inocuos que puedan orientar con mayor seguridad la interacción profesional (56).

El proceso metódico expuesto, adhiere "subrazonamientos" intrínsecos, interdependientes e interactuantes que constituyen un indicador para la evaluación y estudio de los procesos mentales que son ejecutados durante la toma de decisiones en el escenario clínico, así como para el fortalecimiento de su enseñanaza.

El primero y más importante de todos es el razonamiento narrativo donde se entiende al usuario como ser humano único e irrepetible, ya que en él se identifica y comprende la historia de vida, 


\section{"El conocimiento de estos métodos debe ser alimentado utilizado y entrelazado en los transcursos cognitivos y meta- cognitivos de la toma de decisiones..."}

incluidas las prioridades, preocupaciones y estilo de vida del paciente. Para conseguir efectividad y una buena calidad es necesario que el fisioterapeuta desarrolle unas cualidades básicas para la comprensión de la dinámica humana del usuario y la forma como el problema o deficiencia afecta su vida. Estas cualidades hacen referencia a la capacidad de hacer una adecuada organización del conocimiento biosicosocial del usuario, las destrezas comunicativas y la capacidad de apertura del fisioterapeuta en torno a aceptar la perspectiva del usuario frente a su problema $(21,34)$. Este tipo de razonamiento es el eje en torno al cual gira este modelo, ya que permite asegurar las prioridades y necesidades del usuario y se realiza a lo largo del proceso evaluativo.

El Razonamiento interactivo comprende el análisis de la visión de enfermedad/discapacidad del usuario y su familia y los paradigmas que enfrenta en su condición, y requiere de una intervención entretenida y personalizada acorde con los sentimientos y creencias del usuario.

El Razonamiento de Procedimientos se inicia obteniendo información acerca de las dificultades del usuaria para ejecutar determinadas tareas relacionadas con el movimiento corporal humano a través del análisis de las actividades que desempeña, las habilidades necesarias para el desarrollo de la tarea y los ambientes donde se llevan a cabo. En este proceso cognitivo se identifican las conductas de tratamiento y las intervenciones más adecuadas para seguir el plan de acción con base en la evidencia generada por la investigación cualitativa y cuantitativa y por los registros sistemáticos de experiencia profesional.

Razonamiento pragmático: Aquí se identifican las características del entorno socioeconómico, político y normativo que rigen las interacciones profesionales, así como la disponibilidad de recursos técnicos, tecnológicos, financieros y de talento humano, para el aseguramiento de la calidad y efectividad del plan de acción. Se determinan en este nivel los recursos tecnológicos que pueden usarse, las necesidades de remisión a otros profesionales y los criterios para dar de alta a los usuarios.

Razonamiento ético: Se basa en las acciones que deben elegirse de acuerdo con los principios morales y el código ético de la profesión. Se resalta acá la confidencialidad de la información, el manejo de la historia clínica, los deberes y derechos del usuario, el colegaje y las obligaciones y derechos del fisioterapeuta (57.58).

Razonamiento Condicional: Entendido como el auditor perma- nente del proceso de interacción profesional con el fin de dar cum plimiento a las expectativas y demandas del usuario, así como a la: metas establecidas en el plan de acción. Resalta la flexibilidad comc cualidad básica para cambiar o modificar aspectos que puedan in. fluir en el resultado final del tratamiento.

\section{CONCLUSIONES}

Los problemas que los fisioterapeutas clínicos deben afrontar involucran modos y métodos de las ciencias básicas, el consensc profesional, el contexto socioeconómico, normativo y cultural, as: como las bondades y retos derivados de la práctica basada en la evi. dencia y en la experiencia en cada uno de los niveles de interacción. El razonamiento clínico le da el carácter a la disciplina en la medida que potencializa y dinamiza los procesos de toma de decisiones argumentadas y analizadas, basadas en la experiencia y en la evidencia. Este proceso interactivo requiere, por lo tanto, el desarrollo y puesta en uso de competencias cognitivas de alta complejidad (abstracción: análisis, comunicación, observación metódica) que deben construirse a lo largo del proceso de formación profesional y deben seguir afinándose a través de la práctica profesional y la experticia.

El conocimiento de estos métodos debe ser alimentado, utilizado y entrelazado en los transcursos cognitivos y metacognitivos de la toma de decisiones, dinamizando las técnicas de afrontamiento, las propuestas de abordaje, el modelo utilizado y las formas de enseñanza-aprendizaje de la disciplina.

Es importante resaltar la influencia del modelo psicosocial en la comprensión del funcionamiento y la discapacidad(13), por lo cual se requiere considerar todos los factores ambientales, contextuales, personales y culturales que influyen en la salud e implican la aceptación de la relación colaborativa entre el terapeuta, el usuario y su familia, y la comprensión de las necesidades, demandas y expectativas del usuario, así como el asentimiento de las profundas transformaciones que la investigación sobre el razonamiento y la práctica clínica traigan con ella.

Para finalizar, la asertividad del razonamiento clínico depende de la conciencia y precisión de cada uno de sus pasos para identificar problemas susceptibles de interacción que no son observables a primera vista, pero son medibles en la evaluación y revaloración del usuario. 


\section{Referencia: \\ Las referencias a otras obras son una parte muy importante en la literatura científica; ya que estas permiten conocer más sobrę los autores y mantener vivas sus voces dentro del texto.}

1. Rushton, Alison. Moore, Ann. International identification of research priorities for postgraduate theses in musculoskeletal physiotherapy using a modified Delphi technique. Manual Therapy.2010(15) Sup 2: $142-48$.

2. Jones M. Mclntyre I. Naylor S.Are physiotherapy students adequately prepared to successfully gain employment?. Physiotherapy:2010; $96(2): 169-75$.

3. Hunt A, Higgs J Adamson B, L Harris. University Education and the Physiotherapy Professional. Physiotherapy, 1998; $84(6): 26473$.

4. Corral Carlos. El razonamiento médico: Los fundamentos lógicos del método clínico. Ediciones Díaz de Santos, 1994 . P 204.

5. Vargas G. Algunas características epistemológicas de la investigación documental. Revista de Ascolbl. 1988; $1(3-4)$.

6. Amezcua M, Gálvez A. Los modos de análisis en investigación cualitativa en salud: perspectiva crítica y reflexiones en voz alta. Revista Española de Salud Pública. 2002; $76(5): 423-436$.

7. Sánchez-Pedraza R, Gamboa O y Díaz I. Modelos empleados para la Toma de Decisiones en el Cuidado de la Salud. Rev. salud pública 2008(10) sup 1:178-188. Available from: http://www.scielosp. org/scielo.php?script=sci_arttext\&.

8. Conde F. Encuentros y desencuentros entre la perspectiva cualitativa y la cuantitativa en la historia de la medicina. Revista Española de Salud Pública.. 2002: $76(5): 423-436$

9. Real academia española. Diccionario de lengua española. Tomo 2. Ed. Vigésima segunda.200t. p 1293.

10. Peirce C. Reasoning. En: Baldwin JM. Dictionary of Philosophy and Psychology, Smith, Gloucester, MA, 1901, 1925 (reimpresión en 1960). 2001, Traducción castellana de Sara Barrena.

11. Soriguer F. Es la clínica una ciencia?. Ediciones Díaz de Santos, 1 ed. 1992 p 119.

12. Léon Rostan.Curso de Medicina Clinica: con la exposición de los principios de la medicina orgánica o tratado elemental de diagnóstico, pronóstico e indicaciones terapéuticas. Impr. y Libr. de Feros.1839; p 29-36.

13. Foucault M. El nacimiento de la clínica: una arqueología de la mirada médica. Siglo XXI de España Editore.,200\%. p 71-82.
14. International Classification of Functioning, Disablity and Health. Geneva, Switzerland: World Health Organization; 2001.

15. Higgs J. Managing Clinical Education: The Programme Physiotherapy $1993: 79(4): 239-46$

16. Edwards I, Jones M, Hillier S.The interpretation of experience and its relationship to body movement: A clinical reasoning perspective:Manual Therapy 2006 11, Sup 1: 2-10.

17. Jones MA, Jensen G, Edwards I. Clinical reasoning in physiotherapy.In: Higgs J, Jones MA, editors. Clinical reasoning in the health professions 3 th ed. Oxford: Butterworth-Heinemann; 2008. p. 245-56.

18. Titchen A Higgs I. The Nature, Generation and Verification of Knowledge. Physiotherapy $1995: 81(9): 521-31$.

19. Ruiz Ramón. Slence Knowledge, Euler Ruiz, Detroit Michigan (USA) 2000

20. Pasek de Pinto E. disponible en: hitp://diariodelosandesicom/content/ view/21418/105211/.2007.

21. Jones M, Rivett D. Introduction to clinical reasoning. En: clinical reasoning for Manual therapies.1 Ed Elsevier-Sciencie. 2004. P3-24.

22. Brookfield S.Clinical reasoning and generic thinking skills.Clinical reasoning in physiotherapy.In: Higgs J, Jones MA, editors. Clinical reasoning in the health professions.3 thed. Oxford: Butterworth-Heinemann; 2008. p. 245-56.

23. Offredy M, Kendall S, Goodman C.The use of cognitivecontinuum theory and patient scenarios to explore nurse prescribers pharmacological knowledge and decision-making International journal of Nursing Studies 2008 (45):855-68.

24. Bazerman M, Schroth, H, Shah, P, Diekmann, K. The inconsistent role of comparison others and procedural justice in reactions to hypothetical job descriptions: Implications for job acceptance decisions. Organizational Behavior \& Human Decision Processes 1994; 60 (3):326-52.

25. Sladek R, Phillips P, Bond M.Implementation science: a role for parallel dual processing models of reasoning? Disponible en: http://www. implementationscience.com/content/1/1/12. 2006,p 1-8.

26. Facione N, Facione P. Critical thinking and Clinical Judgement. In: Critical thinking and clinical reasoning in the health sciences an international 
multidisciplinary teaching antology.1 ty. Ed. Califonia academic press. 2008. p 1-13.

27. Higss I Jones $\mathrm{M}$, Titchen A. Knowledge, reasoning and evidence for practice. In: Higgs J, Jones MA, editors. Clinical reasoning in the health professions. 3 th ed. Oxford: Butterworth-Heinemann; 2008. p. 245-56.

28. Higgs I, Fish D, Rothwell R. Practicelnowledge-critical appreciation. In:Higgs J, Richardson B, Abrandt M., editors. Developing Practice Knowledge for Health Professionals. 1th. Ed. Elsevier. 2004, P 89-105.

29. Ursua Nicanor. Cerebro y conocimiento: Un enfoque evolucionista. 1 ed. Ed. Anthropos. 1993. P 41-47.

30. Lafuente M. Causalidad y conocimiento según Piaget. Ed. Colegio universitario de León. 1977 p 28.

31. Cañas J, Waerns Y. Engonomía cognitiva. 1 Ed. Médica panamericana 2001. P5-35.

32. Coutinho S, Wiemer-Hastings K, Skowronskij, Britt A. Metacognition, need for cognition and use of explanations during ongoing learning and problem solving. Leaming and Individual Differences 2005:15(4): 321-337.

33. Banning M. Clinical reasoning and its application to nursing: Concepts and research studies. Nurse Education in Practice 2008, 8.( Iss 3):177-183.

34. Higss J, Jones M. Clinical decision making and multiple problem spaces.In: Higgs J, Jones MA, editors. Clinical reasoning in the health professions. 3 th ed. Oxford: Butterworth-Heinemann; 2008. p. 3-17.

35. Cristhensen N, Jones M, Edwards I. Dl razonamiento clínico en el diagnóstico y tratamiento del dolor raquideo en: Grieve Gregory, Boyling Jeffrey, Jull Gwendolen. Terapia manual contemporánea: Columna vertebral 3 ed Blsevier; 2007. P 391-403.

36. Hayes Brett K, Jessamine Chen Tsan-Hsiang. Clinical expertise and reasoning with uncertain categories. Psychonomic Bulletin \& Review 2008, 15 (5): 1002-07

37. Edwards I, Jones M, Hillier S.The interpretation of experience and its relationship to body movement: A clinical reasoning perspective:Manual Therapy $2006,11(1): 2-10$.

38. Hengeveld E, Banks K. Perspectivas contemporáneas en la práctica de la fisioterapia. In: Maitland manipulación periférica. 4 ta. Ed. Elsevier. 2007. p 71-88.

39. Cott C, Finch E, Gasner D, Yoshida K, Thomas S. The movement continuum theory of physical therapy. Physiotherapy Canada $1995(47): 87-95$.

40. Agaméz I, Arenas B, Restrepo H, Toro I, Rodriguez I, Vanegas I y Vidarte J. Sentido del cuerpo para la persona con discapacidad de la locomoción. III informe de avance: El movimiento como sistema complejo. Revista digital efdeportes.com. 2000 Octubre.Año $5(26)$.

41. Beeston S, Simons H. Physiotherapy practice: Practitioners' perspectives. Physiotherapy Theory and Practice 1996 (12): 231-242.

42. Groth G. Clinical Decision Making and TherapistsAutonony in the Context of Flexor Tendon Rehabilitation. H Hand Therapy. 2008(21):254-60.
43. Provincial Rehabilitation Reference Group Managing the Seams:Making the Rehabilitation System Work for People. Toronto, Ontario: Ministry of Health and Long-Term Care, 2000; 1- 67 In: Cott C. Client-centred rehabilitation: client perspectives. Disability and rehabilitation $2004 ; 26,(24): 1411-22$.

44. Cott C. Client-centred rehabilitation: client perspectives. Disability and rehabilitation $2004,26,(24): 1411-22$.

45. Atkins S, Ersser S. Clinical reasoning and patiente-centred care.In: Higgs J, Jones MA, editors. Clinical reasoning in the health professionss thed. Oxford: Butterworth-Heinemann; 2008. p. $77^{-87}$

46. Windish D, Price E, Clever S,Magaziner J, Thomas P. Teaching Medical Students the Important Connection between Communication and Clinical Reasoning Joumal General Internal Medicine 2005(20):1108-13.

47. Edwards I, Jones M, Carr I, Braunack-Mayer A, Jensen G. Clinical Reasoning Strategies in Physical Therapy. Physical Therapy $2004 ; 84(4): 312-30$.

48. Blanco M, Oliva L,Bosch R, Menéndez L, Suárez R. Evaluación de razonamiento dínico. Educ Med Sup 2005; $19(4): 1-7$.

49. Sánchez R, Gamboa O y Díaz J. Modelos empleados para la Toma de Decisiones en el Cuidado de la Salud. Revista de Salud Píblica 2008, 10(1): $178-88$.

50. Teal C, Street R. Critical elements of culturally competent communication in the medical encounter: A review and model. Social Science \& Medicine.2008 $\operatorname{xxxp} 1-11$.

51. Case K, Harrison K, Roskell C. Clinical reasoning process of expert and novice cardiorespiratory physiotherapists. Physiotherapy 2000, 86(1):14-21.

52. Banning $\mathrm{M}$. Clinical reasoning and its application to nursing:Concepts and research studies. Nurse Education in Practice 2008 (8): 177-183.

53. Goodman C, Snyder T. Diferencial diagnosis in Physical therapyz th Ed Saunders. 2000. p $37-87$

54. Rouf E, Chumley H, Dobbie A. Patient-centered interviewing and student performance in a comprehensive clinical skills examination: Is there an association?. Patient Education and Counseling $\mathrm{xxx}$ (2008) xxx-xxx:p 1-5.

55. Realdi G, Previato L, Vitturi N. Selection of diagnostic tests for clinical decision making and translation to a problem oriented medical record. Clinica Chimica Acta. Vol 393.2008 pag $37-43$.

56. Mulligan S. Terapia ocupacional en pediatría proceso de evaluación. Ed. panamericana. 2006. $\mathrm{P}_{3}-6$

57. Confrdence in clinical reasoning.Editorial Physical Therapy in Sport $2008(9): 165-66$

58. Por la cual se reglamenta el ejercicio de la profesión de fisioterapia, se dictan normas en materia de etica profesional y otras disposiciones de 1999. Ley 528. (Septiembre 14-1999). 